\title{
Zur Relevanz wissenschafts-, medizin- und sozialgeschichtlicher Grundlagenforschung*
}

\author{
Josef M. Schmidt \\ Institut für Ethik, Geschichte und Theorie der Medizin, Ludwig-Maximilians-Universität München, Deutschland
}

\section{Schlüssselwörter}

Wissenschaftsgeschichte · Medizingeschichte · Sozialgeschichte . Wirtschaftsgeschichte $\cdot$ Homöopathiegeschichte

\section{Zusammenfassung}

Aus Unkenntnis der eigenen methodischen Grundlagen und Beschränkungen überschreiten Wissenschaftler oft die legitimen Grenzen ihres Faches, wenn sie anderen Wissenschaften deren Wissenschaftlichkeit absprechen. Neue kritische Ansätze in den Natur-, Sozial- und Humanwissenschaften reflektieren hingegen vermehrt die Wechselwirkungen und Interdependenzen zwischen dem Forscher und seinem Fachgebiet bzw. dem Forschungsgegenstand und dem inn selbst erst konstituierenden Rahmen. Aus der Wirtschaftswissenschaft kommt dabei die These, dass das moderne Subjekt und seine Rationalität auf Vergesellschaftungsprozessen in der Denkform des Geldes beruhen, die insofern auch massgeblich das Wesen der modernen Medizin bestimmen. Verglichen mit deren Prinzipien wie Quantifizierung, Standardisierung, Mathematisierung, Reproduzierbarkeit, Materialismus usw. erscheint die Homöopathie, die noch vor dem grossen Aufschwung der Geldwirtschaft im 19. Jahrhundert begründet wurde, als vom monetären Denken relativ freie, nämlich qualitative, individualisierende, hermeneutische und teleologische Medizin.

\section{Keywords}

History of science - History of medicine - Social history . History of economics $\cdot$ History of homeopathy

\section{Summary}

Relevance of Basic Research in the History of Science,

Medicine, and Sociology

Out of ignorance of their own methodological basics and restrictions scientists often trespass the legitimate scope of their discipline when denying other sciences the quality of being scientific. New critical approaches from the natural, social, and human sciences, however, reflect the interactions and interdependencies between the researcher and his field of research or between the subject-matter and its constituting framework. Thus, economic science has provided the thesis that the modern subject and its rationality are based on socialization processes in the form of thinking in terms of money, which also significantly determine the nature of modern medicine. Compared to its principles of quantification, standardization, mathematization, reproducibility, materialism and so forth, homeopathy, which had already been founded before the great boom of money economy took place in the nineteenth century, appears to be relatively free from monetary thinking as a qualitative, individualizing, hermeneutic, and teleological medicine.

\section{Mots-clés}

Histoire de la science $\cdot$ Histoire de la médecine $\cdot$ Histoire sociale . Histoire de l'économie · Histoire de l'homéopathie

\section{Résumé}

Sur la pertinence de la recherche fondamentale en histoire de la science, de la médecine et de l'histoire sociale

La méconnaissance de leurs propres bases méthodiques et de leurs limitations fait que les scientifiques dépassent souvent les frontières légitimes de leur spécialisation lorsqu'ils contestent le caractère scientifique d'autres sciences. Les nouvelles approches critiques des sciences naturelles, sociales et humaines reflètent toutefois de plus en plus les interactions et les interdépendances entre le chercheur et sa spécialisation et/ou l'objet de ses recherches et son cadre constituant. La science économique avance ainsi la thèse que le sujet moderne et sa rationalité se basent sur des processus de socialisation selon la formedepensée del'argent, influençant ainsi demanière déterminante l'esprit de la médecine moderne aussi. Comparée à ces principes comme la quantification, la standardisation, la mathématisation, la reproductibilité, le matérialisme, etc., l'homéopathie, qui avait été fondée avant l'essor de l'économie monétaire au $19^{e}$ siècle, apparait comme relativement libérée de la pensée monétaire, soit une médecinequalitative,individualisante, herméneutiqueettéléologique.

\footnotetext{
*Überarbeitete Version eines Vortrags, gehalten anlässlich des 11. Internationalen Coethener Erfahrungsaustauschs am 10. November $2011 \mathrm{im}$ Veranstaltungszentrum Schloss Köthen, Köthen (Anhalt), Deutschland.
}

\section{KARGER}

Fax +497614520714 Information@Karger.com www.karger.com
Prof. Dr. med. Dr. phil. Josef M. Schmid

Institut für Ethik, Geschichte und Theorie der Medizin

Ludwig-Maximilians-Universität München

Lessingstrasse 2, 80336 München, Deutschland

j.m.schmidt@lrz.uni-muenchen.de 


\section{Einleitung}

Seit ihrer Begründung durch Samuel Hahnemann (17551843) vor etwa 200 Jahren kann die Homöopathie auf viele beeindruckende Errungenschaften zurückblicken, wie unzählige Heilungen von Krankheiten, individuell wie in Epidemien, Popularität bei Millionen von Patienten auf der ganzen Welt, politische Erfolge wie Professionalisierung und Institutionalisierung und wissenschaftliche Forschung - von Einzelfallstudien und klinischen Studien bis hin zu Grundlagenforschung im Labor [1]. Nichtsdestotrotz fehlt es nach wie vor an einer entsprechenden Anerkennung und Würdigung durch die konventionelle Medizin. Die Angriffe auf die Homöopathie scheinen seit der Jahrtausendwende sogar wieder zuzunehmen [2-4].

Wie lässt sich dieses Paradox erklären, wie lässt es sich auflösen? Kommuniziert man aneinander vorbei? Wird wirklich wahrgenommen, was der jeweils andere macht? Sind die bisherigen Forschungsansätze nicht zielführend? Oder bedarf es erst einmal einer medizinhistorischen und medizintheoretischen Revision der Grundlagenforschung, die die Homöopathie vordringlich benötigt?

Im Folgenden wird die These vertreten, dass der Grund, warum die konventionelle Medizin den Anspruch der Homöopathie, eine wissenschaftliche Medizin zu sein, zurückweist, vor allem in einem unzureichenden Verständnis der Reichweite und Grenzen der eigenen Wissenschaft und ihres angemessenen Stellenwerts in unserem Leben liegt. Bei allen Verdiensten und Errungenschaften innerhalb der eigenen Disziplin scheint speziell bei Medizinern ein epistemologisches Bewusstsein ihrer Herkunft, Möglichkeiten und Grenzen nicht immer besonders ausgeprägt zu sein. Wer etwa bei allen - auch sozial- und geisteswissenschaftlichen - Themen nur Fragestellungen zulassen möchte, die auf ein naturwissenschaftliches Format wie Nullhypothese, Datensammlung und kausale Schlussfolgerung reduzierbar sind, ist - trotz der Mächtigkeit und Unentbehrlichkeit der erlernten Methodik im jeweils legitimen Anwendungsgebiet - denkbar schlecht gerüstet, die eigentlichen Probleme der modernen Industriegesellschaft und ihres Gesundheitssystems zu erkennen, zu verstehen oder gar aufzulösen.

In einer Welt, in der wie nie zuvor Menschen samt ihrer Fachwissenschaften Opfer von ökonomisch-physikalischen Theorien, Algorithmen und Ideologien werden [5], ist aber ein einzelwissenschaftliches Pochen auf eingeübte Argumentationsrituale gerade nicht mehr wissenschaftlich im ursprünglichen Sinne, sondern kurzsichtig und kontraproduktiv [6]. Um das Ganze bzw. den Hintergrund von allem zu denken, muss erst die Beschränktheit jedes Einzelnen gesehen werden. Nur so - und nicht durch Rückzug auf Arbeitsteilung, Funktionalismus und Methodenkonsens - können Wissenschaften überhaupt sinnvoll eingesetzt und vor sich selbst, d.h. vor ihrer rationalistischen,
Menschlichem gegenüber indifferenten Eigendynamik, geschützt werden. Subjektlose Wissenschaften bedürfen zur Einschränkung ihres Destruktionspotenzials - um der Menschheit willen - moralischer Subjekte, deren Rationalität ihre Tugend und nicht immer nur Resultat wissenschaftlicher Kalküle ist [7].

Statt in Wissenschaften (einschliesslich der medizinischen) Autoritäten zu sehen, die einen zur Unterwerfung unter ein von ihnen nahegelegtes reduktionistisches Weltbild nötigen, sollten diese vielmehr pragmatisch als Werkzeuge zur Erkenntnis und Praxis benutzt werden. Damit aber nicht - durch Verallgemeinerung und Expansion ihres Einflussbereichs - inhaltlich das rechte Mass überschritten wird, empfiehlt es sich, deren methodisch-kritischen Ansatz immer wieder auf sie selbst anzuwenden und bis zu dem Punkt zu radikalisieren, an dem sie ihre eigenen verborgenen Prämissen und ihre beschränkte Geltung offenlegen müssen. So kann man sich einen frischen und unbefangenen Blick auf die Realität bewahren.

Es wird nun zu zeigen sein, wie die konventionelle Medizin, wenn sie meint, die Homöopathie verurteilen zu können, unwissenschaftlich wird, indem sie erstens über etwas spricht, was ausserhalb ihres methodisch eng begrenzten Gegenstandsbereichs liegt, zweitens in der Reflexion ihrer eigenen epistemologischen Grenzen weit hinter derjenigen anderer Naturwissenschaften zurückbleibt, drittens auch nicht auf dem aktuellen Stand der Diskussion der Theorie der Medizin ist, viertens neuere Ergebnisse der Sozial- und Wirtschaftswissenschaften nicht zur Kenntnis nimmt, und fünftens auch von deren Auswirkungen auf die Humanwissenschaften, bis hin zur medizinischen Ethik, keinen Begriff hat, ebenso wenig wie sechstens von der Geschichte und Theorie der Homöopathie. Dem bis dahin dargelegten, mittels entsprechender Einzelwissenschaften (nur eben nicht mit dem Instrumentarium konventioneller klinischer Medizin) rekonstruierund belegbaren Sachverhalt werden sich zuletzt einige philosophische Überlegungen anschliessen, die zwar nicht im strikten Sinne beweisbar sind, dafür aber eine Möglichkeit bieten, bei aller Kritik und linearen Diskursivität den Kreis am Ende doch wieder zu schliessen.

\section{Geschichte und Theorie der Wissenschaften}

Die sicherste und für Homöopathen sozusagen evidenteste Tatsache ist wohl die, dass «Homöopathen Homöopathie praktizieren». Doch was bedeutet das? Was tun sie dabei? Kann hier überhaupt eine einfache Antwort erwartet werden - oder ist jede Aussage bereits davon abhängig, welche Theorie man zugrunde legt?

Die übliche Einschätzung vonseiten der konventionellen Medizin, der Wissenschaft also, die der Homöopathie von ihrem Gegenstand her am nächsten, von ihrem 
Verständnis her allerdings am fernsten steht, lautet etwa folgendermassen:

- Homöopathen benutzen keine materiellen Arzneisubstanzen, sondern ultramolekulare Verdünnungen.

- Sie verschreiben nicht nach konventionellen Diagnosen, die auf objektivierenden Technologien beruhen, sondern gemäss subjektiver Beschwerden.

- Statt zu versuchen, materielle Krankheitsursachen zu beseitigen, behandeln sie nach dem Ähnlichkeitsprinzip, das nicht als wissenschaftlich im modernen Sinne angesehen werden könne.

Da klinische Studien, die die Goldstandards der evidenzbasierten Medizin erfüllen, wie randomisierte Doppelblindstudien, im Allgemeinen angeblich keinen signifikanten Unterschied zwischen homöopathischer Behandlung und Placebotherapie erkennen lassen [8], zieht die konventionelle Medizin den (Kurz-)Schluss, dass Homöopathen eben Placebotherapie praktizieren.

Diese Aussage kann jedoch nicht als wissenschaftlich erachtet werden - solange sie nämlich ihre eigenen Voraussetzungen und ihre sie begrenzenden Rahmenbedingungen verschweigt. Denn tatsächlich erscheint jeder der genannten Punkte nur dann kritisierbar, wenn man sich auf einen ganz bestimmten Standpunkt innerhalb eines sehr beschränkten Horizonts stellt, was allerdings nicht notwendig ist. Richtig müsste die Aussage der quantifizierenden konventionellen Naturwissenschaft vielmehr lauten: Unter der Voraussetzung eines naiven Materialismus und ausschliesslich quantitativ-statistischer Methodik lässt die bisher erhobene Datenlage keinen zwingenden Schluss darauf zu, dass damit die Homöopathie oder auch Placebotherapie angemessen erfasst, verstanden und beurteilt wäre, da beide möglicherweise Phänomene sind, die sich dem konventionellen Set naturwissenschaftlichmedizinischer Kategorien entziehen.

Die Zurückweisung unbegründeter Ansprüche und Behauptungen vonseiten der konventionellen Medizin sollte freilich nicht missverstanden werden als eine Ablehnung des modernen wissenschaftlichen Ansatzes überhaupt. Die Methode des systematischen Beobachtens, Messens und Experimentierens und die Formulierung, Testung und Modifikation von Hypothesen, wie sie erfolgreich in der Physik, Chemie und auch in der Medizin angewandt wird, hat sich als sehr erfolgreich erwiesen, und niemand möchte auf ihre Errungenschaften verzichten.

Das einzige, aber entscheidende Problem ist, dass die damit erreichten Annehmlichkeiten durch eine dunkle, für die Menschheit bedrohliche Seite unterminiert werden, wenn darüber die nur instrumentelle Funktion der Wissenschaft vergessen wird und die ganze Welt und unser Leben nichts anderes mehr sein sollten als das, was Wissenschaftler messen und in Tabellen demonstrieren können.
In der Quantenphysik, einem der durchdachtesten und konsequentesten Zweige der Naturwissenschaften, ist der naturwissenschaftliche Ansatz seit langem an einen Punkt gelangt, wo fundamentale Voraussetzungen der modernen Naturwissenschaft, ihr naiver Realismus, Objektivismus und Materialismus, sich als unhaltbar erwiesen haben. Statt weiter zu hoffen, herauszufinden, ob die «Realität» aus Teilchen oder Wellen besteht, können Wissenschaftler zeigen, dass der Messakt weniger vermeintlich objektive Entitäten aufdeckt, als vielmehr zu einem Kollaps des Systems als Ganzem führt und letztlich nur ein menschengemachtes Bild der Welt konstituiert und fixiert. Wenn der Wissenschaftler der Natur eine Teilchen-Frage stellt, liefert sie ihm Teilchen-Daten. Wenn er eine Wellen-Frage stellt, wird ihre Wellen-Antwort ihn zur Konstruktion eines wellenbasierten Weltbildes veranlassen usw. [9].

Die gleiche wissenschaftliche Einsicht, die die konventionelle Naturwissenschaft relativiert und ihr den ihr zukommenden Platz anweist, ist in der modernen Biologie zu finden - in Form des radikalen Konstruktivismus. Wahrnehmung wird hier nicht mehr als passives Aufnehmen von Daten angesehen, die durch die Sinne von einer vermeintlich objektiven Welt draussen zu uns herein gelangen, sondern eher als Konstruktionsprozess durch das Subjekt, auf dessen Veranlagung, Interesse, Geschichte usw. es daher mehr ankomme als auf die Übereinstimmung mit einer vermeintlich objektiven Aussenwelt. Dementsprechend werden dogmatische Konzepte wie Wahrheit, Realität oder Objektivität hier durch das pragmatische Kriterium der Viabilität (Gangbarkeit) ersetzt, d.h. den Test, ob eine Idee oder ein Konzept sich in der Praxis bewährt oder nicht [10].

Die moderne Wissenschaftstheorie befasst sich mit diesen Fragen auf eine allgemeinere, doch ebenso kompromisslose Art. Nachdem Positivismus, kritischer Rationalismus (Popper) und genereller Methodenzwang wirksam zurückgewiesen wurden (Feyerabend), bewies z.B. in der Mathematik die Aufstellung des UnabschliessbarkeitsTheorems die inhärente Begrenztheit aller axiomatischen Systeme (Gödel), und in der philosophischen Logik konnte gezeigt werden, dass jede Wissenschaft auf absoluten Voraussetzungen beruht, die aus ihr selbst nicht rational ableitbar sind (Collingwood). Aufbauend auf der Herausarbeitung von «Denkstilen» und «Denkkollektiven», die jeder wissenschaftlichen Tatsache zugrunde liegen (Fleck), ist es in der Epistemologie inzwischen weitgehend anerkannt, dass Wissenschaft wesentlich ein sozialer Prozess ist, der erfolgreich innerhalb bestimmter Paradigmen arbeitet, die allerdings keine absolute Gültigkeit haben, sondern von Zeit zu Zeit geändert werden können - und in der Tat geändert wurden, wie die Wissenschaftsgeschichte zeigt (Kuhn) [11]. 


\section{Geschichte und Theorie der Medizin}

Die Theorie der Medizin hat sich, inspiriert von diesen Einsichten, seit der zweiten Hälfte des vergangenen Jahrhunderts als eigene Disziplin herausgebildet und beachtlich entwickelt. Im Gegensatz zur herkömmlichen Einschätzung der Medizin als nichts als eine angewandte Naturwissenschaft wird es nunmehr für zwingend gehalten, die Medizin für eine praktische Wissenschaft sui generis zu erachten - ganz in Übereinstimmung mit dem traditionellen Konzept der Heilkunst. Da Medizin primär von der Verpflichtung des Arztes ausgeht, dem Patienten zu helfen, d.h. von seiner Pflicht zu handeln oder Ratschläge zu erteilen, kann ein blosses Ansammeln von Wissen hier niemals ein abgetrenntes Ziel in sich selbst sein, sondern immer nur sekundären Status haben als ein Mittel, das primäre Ziel wohltätigen Handelns zu erleichtern [12].

Neben der Analyse ihres gegenwärtigen Status hat die Theorie der Medizin auch konkrete Modelle entwickelt, um ihren Ansatz und das Verständnis ihres genuinen Objekts, des Menschen, zu erweitern. Zu diesem Zweck wurde vorgeschlagen, das Konzept der Subjektivität, also das menschliche Subjekt, in die Medizin einzuführen [13]. Entlang dieser neuen Denklinien wurde unter anderem das biopsychosoziale Modell des Menschen als eine Möglichkeit ausgearbeitet, den Patienten als eine Einheit physiologischer, mentaler und sozialer Ebenen der Existenz sowie als eine Einheit eines menschlichen Subjekts und seiner individuellen Umwelt wahrzunehmen und zu behandeln [14]. Anders als bei der konventionellen Verdinglichung des Menschen als mechanistische physikalisch-chemische Maschine werden hier Leben, Krankheit und Heilung des Patienten im Sinne von Bedeutungsprozessen, Funktionskreisen und Situationskreisen verstanden.

Mithilfe dieses dynamischen Modells des Menschen, das heute in der Medizintheorie als eines der ausgefeiltesten und konsistentesten gilt [15], kann die Praxis der Homöopathie auf eine angemessene und erhellende Weise beschrieben und verstanden werden. Anstatt materielle Entitäten wie menschliche Körper, kranke Organe oder Arzneisubstanzen als Basis für das Denken zu nehmen, kann der funktionale, kybernetische und semiotische $\mathrm{Zu}$ gang zu den Phänomenen des Lebens, der hier vertreten wird, die Patienten als Lebewesen anerkennen, die ihre Welt und ihren aktuellen Zustand jeweils durch den Vollzug von Bedeutungserteilungs- und Bedeutungsverarbeitungsprozessen selbst erzeugen. Wenn z.B. ein Patient in einem Zustand ist, der nach einem bestimmten Mittel verlangt, bietet der Arzt, der dieses besondere Verlangen mittels einer semiotischen Interpretation der Symptome herausgefunden hat, dieses Mittel dem Patienten an, der, indem er einen Funktionskreis der Verarbeitung der Arznei-Information durchläuft, sich selbst heilt. Nicht das
Arzneimittel heilt also den Patienten, sondern der Patient heilt sich selbst (mithilfe des Mittels), zumal er selbst schliesslich der einzig lebendige Akteur in diesem Prozess ist. Und nicht die Krankheit oder die Symptome des Patienten determinieren das richtige Mittel, sondern der Arzt bestimmt es, weil Handlungen, wie das Finden oder Auswählen eines Mittels, nur von menschlichen Lebewesen vollzogen werden können.

Der wichtigste Unterschied dieses stimmigsten und aktuellsten Modells des Menschen [15] im Vergleich zum konventionellen materialistischen Modell ist, dass es das, was Homöopathen tun, auf eine wissenschaftliche Weise repräsentieren und erklären kann, ohne ständig von materialistischen Einwänden und Skeptizismen behindert zu sein. Wenn Bedeutungsprozesse in der Welt des Lebendigen primär und konstitutiv sind, scheint es sekundär zu sein, ob eine vom Patienten wahrgenommene und verarbeitete medizinische Information physisch, chemisch oder virtuell ist, solange sie genau die Bedeutung repräsentiert, auf die er in seinem momentanen Zustand fixiert ist. Darüber hinaus bieten die Begriffe und Konzepte, wie sie im biopsychosozialen Modell verwendet werden, Homöopathen ein Instrumentarium zur wissenschaftlichen Klärung und Spezifikation des eigenen Selbstverständnisses [16].

\section{Geschichte und Theorie der Sozialwissenschaften}

Wie kommt es aber, dass diese fortschrittlichste und wissenschaftlichste Sicht des Menschen [15] in der medizinischen Gemeinschaft bei Weitem nicht so bekannt und verbreitet ist wie die konventionelle, vergleichsweise triviale, materialistische Sichtweise? Um dieses zweite Paradox zu beantworten, müssen für einen Moment die Naturwissenschaften verlassen und auch die Sozial- und Geisteswissenschaften, die sogenannten Lebenswissenschaften, berücksichtigt werden.

Aus Sicht der Soziologie sind Menschen soziale Wesen, d.h., sie schliessen sich zusammen und vergesellschaften sich, bilden also Gesellschaften, auf eine spezifische Weise. Während die konventionelle Soziologie Gesellschaften meist untersuchte, als ob sie neutrale Forschungsobjekte von davon unabhängigen wissenschaftlichen Beobachtern wären, betonen neuere kritische Ansätze die gegenseitige Interdependenz zwischen individuellen Subjekten und der Gesellschaft als Ganzes. Da eine Gesellschaft nicht eine Aggregation materieller Dinge ist, sondern das Ergebnis mentaler Bedeutungsprozesse, die sowohl individuell als auch kollektiv vollzogen werden, hängt ihre Form und Existenz von ihrem eigenen Rekonstruktionsprozess durch ihre Subjekte ab, die wiederum durch eine permanente kollektive Konstruktion und Aufrechterhaltung von Bedeutungen konstituiert und geprägt werden [17]. 
Ein Beispiel aus der Kommunikationswissenschaft möge diesen Punkt etwas klarer machen. Lässt man den konventionellen linguistischen Ansatz und seine Analyse von Worten und Sätzen, als ob dies objektive Entitäten wären, hinter sich, so erscheint, aus einer kritischen Perspektive, Sprache als ein soziales Phänomen. Sie braucht Intersubjektivität, d.h. Dialogpartner, und kann daher nicht von einer solipsistischen Person auf einer einsamen Insel geschaffen oder entwickelt werden. Auch ein Eremit kann nur deshalb mit sich selbst oder mit seinem Gott sprechen, weil er vorher in einem sozialen Rahmen überhaupt sprechen gelernt hat: im Kontakt mit seiner Mutter, Familie usw. Wir sind in unsere Sprache hineingeboren, sie ist primär und wir (als sie Sprechende) sind sekundär. Auf diese Weise konstituiert die vorgefundene Sprache uns als Muttersprachler des Deutschen, Englischen usw. Sprache ist ein Bedeutungsprozess, dessen Dasein und Gegenwart vom Vollzug und von der Teilnahme der Sprecher abhängt. Um andererseits zu verstehen, was Sprache ist, kann man sich ihr nicht von aussen nähern, von einer vermeintlich neutralen Position, sondern man muss an ihr teilnehmen.

Auf die Homöopathie angewandt, legt dieses Zwischenergebnis aus den Sozialwissenschaften die Schlussfolgerung nahe, dass analog die Homöopathie nicht ohne Teilnahme an ihr, d.h., ohne sie wirklich zu praktizieren, verstanden werden kann.

Wenn Sprache, Wissenschaft und Homöopathie allerdings soziale Bedeutungsprozesse sind, stellt sich nichtsdestotrotz die Frage: Welche Art von Bedeutung wird hier verarbeitet, und auf welche Art und Weise?

\section{Geschichte und Theorie der Wirtschaftswissenschaft}

Auf den ersten Blick scheint die Wirtschaftswissenschaft nichts mit diesen Fragen zu tun zu haben. Bei tieferer Betrachtung ergibt sich allerdings, dass die Ökonomie einen enormen Einfluss auf praktisch alle Bereiche unseres Lebens hat - von der Weise, wie wir uns sehen, bis zu der Weise, wie wir unsere Wissenschaften betreiben.

Konventionelle Wirtschaftswissenschaft, wie sie gewöhnlich an Universitäten und Wirtschaftsschulen gelehrt wird, beansprucht für sich, Wirtschaft im Sinne von Güterknappheit, Sachzwängen und Geld als Tauschmittel zu erklären - als ob Waren, Kaufleute und Märkte seit jeher immer schon existiert hätten. Egoistischer Eigennutz wird hier nicht hinterfragt, sondern vorausgesetzt und dadurch gebilligt, während ökonomische Probleme auf eine Art und Weise untersucht und behandelt werden, wie ein Ingenieur einen Mechanismus, der unabhängig von ihm selbst abläuft, analysieren und reparieren würde. Diese vermeintlich objektivistische Sicht ist freilich nur scheinbar objektiv [18]. Sie scheint vielmehr dem Zweck einer Rechtfertigung freier Märkte zu dienen, ebenso wie die Ideologie, die zuerst von Adam Smith (1723-1790) vorgeschlagen wurde, der zufolge eine Art «unsichtbare Hand» die Auswirkungen eigennütziger ökonomischer Akteure in den Wohlstand der Nationen verwandeln soll [19]. Mittlerweile haben Wirtschafts- und Finanzkrisen auf der ganzen Welt hinreichend gezeigt, dass dies so nicht der Fall ist. Die Theorie einer «Oikodizee» der Märkte (in Analogie zur Theodizee Gottes) gilt heute als gescheitert [20].

Versucht man hingegen, der Sache auf den Grund zu gehen, ergibt sich, dass Geld nicht ein Ding, eine Substanz oder etwas ist, das einen intrinsischen Wert hat, sondern lediglich eine Denkform, die Weise der Vergesellschaftung in modernen kapitalistischen Gesellschaften. So wie wir uns immer schon in der in unserem Land gesprochenen Sprache vorfinden, so befinden wir uns auch immer schon in einer spezifischen Form des Gelddenkens. So wie wir nicht sprechen lernen können, ohne an der in unserer frühkindlichen Welt gesprochenen Sprache teilzunehmen und sie dadurch als unsere Muttersprache zu akzeptieren, so können wir auch nicht denken und rechnen lernen, ohne an der Logik des Geldes als dem Ursprung unserer logischen Grundkategorien teilzunehmen und sie damit zu akzeptieren [21].

Zusammen mit den Einsichten der kritischen Soziologie, der Wissenschaftstheorie, des Konstruktivismus usw. bedeutet dies, dass unsere Sicht der Welt, in der wir leben, hauptsächlich durch unser Denken in der Geldform konstituiert ist, da alle unsere Denkprozesse immer schon von Geld als der dominierenden Denkform geprägt sind. Nur weil Geld nichts als eine abstrakte Form des Denkens ist, kann es tatsächlich alles, was es berührt, in eine Ware verwandeln. Im Gegensatz zu Lebewesen oder körperlichen Dingen ist Geld frei von allen Attributen, ist rein abstrakte Quantität, kann deshalb aber auch durch geschickten Handel oder über Zinsen multipliziert werden. Während natürliche Ressourcen begrenzt, d.h. nicht unendlich vermehrbar sind, lässt sich Geld ins Unendliche vervielfältigen und übt in der Tat eine entsprechende inhärente Versuchung dazu aus - vergleichbar etwa einem Virus.

Daher ist in kapitalistischen Zivilisationen die fundamentale Intention, die alle Bereiche des Lebens und der Kultur durchdringt, das höchste Motiv, Ziel und Verdienst der Menschen der Umsatz und die Vermehrung von Geld, genannt Wirtschaftswachstum. Unter diesem Vorzeichen kann alles und jeder als potenzielles Mittel zur finanziellen Bereicherung angesehen werden. Dies ist der Grund, warum Ärzte und pharmazeutische Unternehmen (ob sie wollen oder nicht) Patienten als Mittel zum eigenen Gelderwerb benutzen, warum von Wissenschaftlern (ob sie wollen oder nicht) erwartet wird, Ergebnisse zu liefern, die den Interessen ihrer Sponsoren nicht zuwider laufen, und warum laufend Bedürfnisse nach neuen Produkten 
erzeugt werden, seien diese medizinisch sinnvoll oder nicht - mit dem leitenden Ziel, neue Kunden zu akquirieren, seien diese krank oder gesund [22]. Genuin ärztliche Haltungen und Werte sowie das darauf gegründete Vertrauensverhältnis geraten dabei leicht unter die Räder [23]. Tatsächlich gilt das Bruttoinlandsprodukt, d.h. das Ausmass der Monetarisierung möglichst vieler Bereiche des Lebens, als Indikator für den Lebensstandard in einem Land. Es ist jedoch nur ein quantitatives Mass, das alle qualitativen Dimensionen, die das Leben bieten mag, ignoriert.

Die Vorherrschaft des Geldes ist aber nicht unvermeidbar und auch keine anthropologische Konstante. Die Geschichtswissenschaft kann zeigen, dass dieser Zustand nicht immer gegeben war, wenigstens nicht im heutigen Ausmass. In der konventionellen Historiographie wird Geldgier zwar jeder Epoche gleichermassen unterstellt. Mithilfe eines komparatistischen Ansatzes, der auch die Wirtschaftsgeschichte umfasst, wird dagegen deutlich, wie viele grundlegende und dramatische kulturelle und wissenschaftliche Veränderungen sich in strikter Korrelation mit dem Aufstieg des modernen Gelddenkens während der letzten Jahrhunderte vollzogen haben und sich weiterhin vollziehen [24].

Grob gesagt waren im Mittelalter Agrar- und Subsistenzwirtschaft vorherrschend, und Geld, in Form von Gold- oder Silbermünzen, spielte keine prominente Rolle. Die Mehrzahl der Menschen lebte, arbeitete, ass und trank und half sich gegenseitig hauptsächlich auf der Basis christlicher Werte und ohne Zwischenschaltung von Geld oder finanzieller Berechnung zwischen ihre Handlungen. Kranke und Invalide wurden in ihren Familien oder in Hospizen von Bruderschaften gepflegt, und Heiler wurden, soweit verfügbar, in Naturalien bezahlt. Sobald jedoch die ersten Börsen gegründet und Banknoten gedruckt waren, kam eine bis dahin unbekannte Ruhelosigkeit, Aufgeregtheit und Dynamik auf, hervorgerufen durch den neuen Anreiz, durch Knüpfung und Ausweitung von Handelsbeziehungen Geld und Vermögen zu vermehren. Dies führte zur Entdeckung und Eroberung neuer Kontinente, Kolonisierung und Sklaverei sowie zu Naturausbeutung, Umweltverschmutzung usw. [25].

Im Zuge dieser grundlegenden Veränderungen des Lebens, Strebens und Urteilens, die durch den neuen Stellenwert des Geldes als vorherrschende Denkform ausgelöst wurden, entstand auch die moderne Naturwissenschaft, die sich expressis verbis vom traditionellen teleologischen Denken lossagte und emanzipierte. Seit Francis Bacon (1561-1626) besteht die grundsätzliche Haltung des modernen Wissenschaftlers gegenüber der Natur nicht mehr in Respekt und dem Wunsch, mit ihr in Harmonie zu leben, sondern in der Versuchung, ihr ihre Geheimnisse (mit Schrauben und Zwingen) zu entreissen und sie $\mathrm{zu}$ beherrschen [26] - weil eben Geld verdient werden kann mit Erfindungen, zu denen ein Wissen nötig ist, das auf diese Weise gewonnen wird. Quantifizierung, Mathematisierung, Standardisierung, Reproduzierbarkeit, Materialismus, Positivismus, Reduktionismus usw., d.h. Konzepte, auf die die konventionelle moderne Naturwissenschaft und seit dem 19. Jahrhundert auch die konventionelle moderne Medizin wesentlich gegründet sind, würden keinen Sinn ergeben ohne den Kontext des Vergesellschaftungsprozesses durch das Gelddenken in modernen kapitalistischen Gesellschaften. Indigenen Kulturen müssen diese Konzepte heute noch absurd erscheinen.

\section{Geschichte und Theorie der Humanwissenschaften}

Eine weitere Nebenwirkung der Herrschaft des Geldes als Denkform war die Beschleunigung und Konzentration aller Aktivitäten im Leben. Dies lässt sich in den Kulturwissenschaften zeigen, vorausgesetzt, sie werden kritisch betrieben. Nachdem das mittelalterliche Zinsverbot ausgehöhlt und schliesslich abgeschafft worden war, stieg die Bedeutung der Zeit - als ein Faktor zum Erwerb (oder Verlust) von Geld durch Zinsen - in der öffentlichen Aufmerksamkeit. Schliesslich wurden Zeit und Geld sogar gleichgesetzt. Turm- und Taschenuhren waren bald allgegenwärtig, physiologische Zeit wurde durch Chronometrie ersetzt, und Uhrwerke wurden zum Paradigma jeder Art von wissenschaftlichem Mechanismus. Abgesehen vom Raum kann auch die Zeit ausgebeutet werden, indem man sie mit immer neuen Möglichkeiten, Geld zu verdienen, überfrachtet. In Zivilisationen, in denen Konsumenten weitgehend mit dauerhaften materiellen Gütern, wie Kühlschränken, Autos oder Fernsehgeräten, gesättigt sind, drängen die Märkte die Menschen zunehmend dazu, immer mehr in immer weniger Zeit zu konsumieren. Zum Beispiel, gleichzeitig zu telefonieren, einen Computer zu bedienen, Musik zu hören, Bahn zu fahren, Zeitung zu lesen, einen Kaffee zu trinken usw. [27]. Diese Art von Zeitverdichtung, bekannt unter dem Euphemismus «Multitasking», ist ein direktes Ergebnis der Herrschaft des Geldes in kapitalistischen Zivilisationen. Mittlerweile haben deren unerwünschte Auswirkungen auch die medizinischen Fakultäten und ärztlichen Praxen erreicht [28]. Das Laufrad dreht sich auch hier immer schneller [29].

Nicht einmal die Wissenschaft der Philosophie, d.h. die Selbstreflexion des Geistes, ist frei vom Einfluss des Geldes als Denkform. Überschreitet man die konventionelle Philosophie, die sich meist auf Philosophiegeschichte beschränkt, wird man bei einem kritischen, radikalisierten Ansatz entdecken, dass der Begriff «ratio» von der kaufmännischen Rechnungslegung in der römischen Antike stammt. Überdies lässt sich zeigen, dass sowohl unser Identitätsbewusstsein als auch unsere Rationalität zeitgleich mit dem Aufkommen und der Ausbrei- 
tung der Geldwirtschaft in der Antike entstanden sind [30]. Mit dieser Einsicht erscheint allerdings der Begriff Rationalismus, samt seinen Begleiterscheinungen Aufklärung, Fortschritt, Emanzipation usw., möglicherweise in einem anderen Licht. Um von Fortschritt zu sprechen, müsste man eigentlich wissen, wohin man schreiten will bzw. was erstrebenswert ist. In einer Welt, die von Geld als vorherrschender Denkform dominiert ist, wäre die nächstliegende Antwort: Gut ist, was zu Wirtschaftswachstum führt. Andere Dimensionen des Lebens könnten hier leicht übersehen werden. Seit dem 19. Jahrhundert sind Philosophen zunehmend Konzepten von Dynamik, Fortschritt, Steigerung usw. aufgesessen, ohne zu bemerken, dass sie selbst dabei eher die Rolle eines Sprachrohrs des Zeitgeistes als die des Schöpfers einer eigenständigen Philosophie einnahmen.

Auch der moderne Begriff der Autonomie entpuppt sich in diesem Zusammenhang als eine Täuschung des modernen Subjekts, das spätestens dann konstituiert wurde, als René Descartes (1596-1650) die Formel prägte: «ego cogito, ergo sum» (ich denke, also bin ich) [31]. Zu einer Zeit, als die Menschen ihre Hoffnungen vermehrt auf Geld statt auf persönliche Beziehungen zu ihren Mitmenschen setzten, war seit den ersten Börsen-Crashs das Vertrauen in den abstrakten Wert des Geldes immer gemischt mit der Angst um dessen Verlust. Dieser existenzielle Zweifel, der die gesamte Neuzeit in einer zunehmenden Weise durchzieht, war Descartes' Ausgangspunkt. Seine Schlussfolgerung, die vermeintliche Gewissheit seiner Subjektivität, erscheint eher als Selbsttäuschung, da sein vermeintlich autonomes Ego weiterhin abhängig war von sozialen Prozessen wie Sprechen, Denken und Rechnen in der Geldform. Tatsächlich ist der Begriff eines auf sich selbst zurückgeworfenen abstrakten Subjekts das Resultat eines Denkprozesses in der Geldform und deren inhärenter Unsicherheit.

Sogar die Wissenschaft der Ethik scheint von der Rationalität quantifizierenden und kompetitiven Gelddenkens durchdrungen zu sein. Seit dem 18. Jahrhundert wird beispielsweise im Utilitarismus argumentiert, eine Handlung sei dann ethisch, wenn sie den Gesamtnutzen der grössten Zahl von Individuen maximiere. Seit dem 19. Jahrhundert wurde im Sozialdarwinismus der Begriff des Lebenskampfes, wie er auf kapitalistischen Märkten zu beobachten ist, in das Tierreich projiziert, um ihn schliesslich von dort wieder abzuleiten und damit unsoziale Politik zu rechtfertigen. In der modernen medizinischen Ethik gibt es die Empfehlung, in problematischen Situationen die Interessen aller Beteiligten (ökonomische, finanzielle, soziale, familiäre Interessen) untereinander auszuhandeln gemäss dem Leitparadigma des Verhandelns auf dem Marktplatz. Als Entscheidungshilfe werden dabei nur noch sogenannte mittlere Prinzipien wie Nichtschaden, Wohltun, Respekt vor der Autonomie des Patienten sowie Gerechtigkeit anerkannt [32]. In einem solchen Rahmen kann es allerdings leicht passieren, dass menschliche Dimensionen, wie Liebe, Glaube, Pflicht, Schuld, Scham, Tugend usw., nur noch als Faktoren unter anderen wahrgenommen und damit eigentlich missverstanden und letztlich entwertet werden $[33,34]$.

Schliesslich ist auch in der Theologie zu sehen, wie sich die globale Entwicklung widerspiegelt. Die traditionelle christliche Vorstellung von Gott als einer ewigen, unveränderlichen Substanz oder Wesenheit war bis ins Mittelalter völlig plausibel, solange eben das Leben auf Erden sich daran orientierte und damit übereinstimmte. Nach ökonomischen Sündenfällen wie dem Ablasshandel und einer zunehmenden Rationalisierung der Religion seit der Reformation, im Zuge der Industrialisierung und Beschleunigung des Lebens, ging die Beschaulichkeit des Lebens verloren und konkurrierende individuelle Interessen erforderten eine neue bürgerliche Moral, die auch Strategien, auf gnadenlosen Märkten überleben zu können, umfasste, wie etwa Heuchelei, Scheinheiligkeit und Täuschung. Der Begriff der Wahrheit sowie dessen Garant, Gott, wurden dadurch herausgefordert und letztlich aufgegeben. Friedrich Nietzsche (1844-1900), der Seismograph bürgerlicher Unaufrichtigkeit und Prophet des heraufkommenden Nihilismus des 19. Jahrhunderts, schrie die schauerliche Diagnose hinaus: «Gott ist tot ... und wir haben ihn getötet!» [35].

Um all diese Einsichten verschiedener Wissenschaften $\mathrm{zu}$ einer Schlussfolgerung zu vereinen, kann man sagen, dass in der Neuzeit, besonders in der Moderne, der Prozess der Vergesellschaftung in der Denkform des Geldes in kapitalistischen Zivilisationen alle Bereiche und Dimensionen des Lebens gewaltig verwandelt hat, einschliesslich sämtlicher Wissenschaften. Dies geschah auf eine Weise, dass heute vonseiten der Naturwissenschaften, einschliesslich der Medizin, offenbar nur noch materielle Dinge und quantitative, ausbeutbare Beziehungen als real akzeptiert werden, während alles andere der Gefahr unterliegt, vernachlässigt und geringgeschätzt zu werden - wie etwa Qualitäten, Werte, Gefühle oder eben Bedeutungsprozesse, die mit diesen Kategorien nicht erfassbar sind.

Dies ist der Grund, warum in der konventionellen Medizin das biopsychosoziale Modell des Menschen keine Chance hat, eine signifikante Rolle zu spielen, und warum die Homöopathie immer noch von einem materialistischmechanistischen Hintergrund aus - mittels Statistik und Messdaten - bewertet wird.

\section{Geschichte und Theorie der Homöopathie}

An dieser Stelle erhebt sich als drittes Paradox die Frage, wie es denn möglich sei, den allumfassenden Einfluss des Geldes als Denkform sowohl zu erkennen als auch sich 
davon zu befreien, wenn dieser bereits jedermanns Geist und Logik infiltriert haben sollte.

Nun sind die Homöopathen herausgefordert, sich zu fragen: «Was bedeutet es für mich, Homöopathie zu praktizieren?» Tatsächlich ergeben sich von hier aus weitere, wesentliche Fragen wie: «Was mache ich eigentlich?» und: «Wer bin ich überhaupt?»

Hier mag die Geschichte der Homöopathie den entscheidenden Schlüssel bieten [36]. Während die konventionelle moderne Medizin durch eine Vielzahl sozioökonomischer Bedingungen, Anreize und Interessen unterschiedlichster Akteure im Verlauf von zwei Jahrhunderten konstituiert ist, hat die Homöopathie nur einen Begründer, Samuel Hahnemann (1755-1843). Indem dieser noch in einer Zeit lebte, bevor der gewaltige Einfluss des Rationalismus in der Denkform des Geldes alle westlichen Gesellschaften, Kulturen und Wissenschaften, besonders die moderne Medizin, infiltrierte, haben Homöopathen die Möglichkeit, sich Hahnemanns ökonomisch und moralisch vergleichsweise unkorrumpiertes Leben und Wirken zum Vorbild zu nehmen und von dieser (historisch distanzierten) Warte aus Missstände der Gegenwart leichter zu erkennen. Während heutige Mediziner von Kindheit an zu einer Denkweise sozialisiert werden, die die Expansion von Geldmärkten eher gewährleistet als das Finden von so etwas wie Wahrheit, ging es Hahnemann genau um letztere, zum Wohl seiner «Menschenbrüder» [37].

Hahnemann war freilich kein Heiliger. Geboren am Schnittpunkt zweier ungleicher Epochen, war er hin und her gerissen zwischen einem konservativen metaphysischen Glauben an Gott, Wahrheit, Moral, Vorsehung, vorurteilsfreier Beobachtung usw. einerseits und progressiven wissenschaftlichen Ambitionen, eine rationale Heilmethode zu begründen, mit der Krankheiten mit mathematischer Gewissheit zu heilen seien, andererseits [38]. Nach einem vorübergehenden rationalistischen Überschwang fand er $1819 \mathrm{zu}$ einem ausgewogenen Konzept der Heilkunst. Zur gleichen Zeit bemerkte er aber auch, dass ein zunehmender Anteil der Bevölkerung nicht mehr gesund war, sondern sich im Zustand einer chronischen Krankheit befand. 1828 interpretierte er diese Beobachtungen (innerhalb des ihm zur Verfügung stehenden medizinischen Begriffssystems) als Infektion mit einem chronischen Miasma: Psora, Sykosis oder Syphilis [39]. Interessanterweise war dies eben die Zeit, als sich die gesundheitsschädigenden Effekte der Industrialisierung, Beschleunigung und Monetarisierung zunehmend auch in Sachsen-Anhalt (Leipzig, Köthen) sowie bei Hahnemanns korrespondierenden Patienten in anderen Ländern bemerkbar machten.

Wenn konventionelle Ärzte heute von nichtmedizinischen Akteuren wie Labor-Wissenschaftlern, Pharmareferenten, Juristen, Ökonomen, Politikern usw. instruiert werden, was sie zu tun und zu lassen haben, erscheinen ihre Entscheidungen weitgehend fremdbestimmt durch monetäre Interessen [40]. Als Gegengewicht dazu können Homöopathen heute immer noch von Hahnemann lernen, wie es war bzw. wie es wäre, wenn ein Arzt wagt, selbst zu denken und aus eigenem Antrieb zu handeln, allein unter der Gewähr seines Gewissens und seines Glaubens - um sich dadurch wirksam gegen die Übermacht moderner Formen des Gelddenkens zu immunisieren.

Hahnemann war zwar durchaus mit Geld vertraut. Nachdem er als junger Arzt und Familienvater unter dessen Mangel gelitten hatte, kam er in seinen späten Jahren, unter anderem durch ein innovatives Abrechnungssystem mit Barzahlung im Voraus, sogar zu einigem Wohlstand, sodass er seinen Töchtern 1835, als er nach Paris ging, in Köthen zwei Häuser und einige Tausend Taler vermachen konnte [41]. Sein Umgang mit Geld war aber auf den notwendigen täglichen Gebrauch beschränkt, im Sinne von Aristoteles' Begriff der besonnenen «oikonomía», ohne dass sein Weltbild oder Denken dadurch massgeblich determiniert worden wäre. Nie sah er Geld als Selbstzweck, als Mittel zum Gelderwerb als solchem an, im Sinne von Aristoteles' Gegenbegriff der masslosen «chremastiké» [42].

Das Entscheidende hier ist, dass Hahnemanns Lehre als solche unbelastet ist vom Denken in der Geldform, also noch frei von Konzepten wie Quantifizierung, Standardisierung, Mathematisierung, Reproduzierbarkeit, Materialismus, Mechanismus, Statistik usw. Im Gegenteil, sein Ansatz ist dezidiert qualitativ, individualisierend, hermeneutisch, teleologisch und voller Respekt für Eigentümlichkeiten und Details der einzelnen Patienten. Homöopathen wären gut beraten, dieses Erbe eher als Privileg und Herausforderung denn als peinlichen Anachronismus zu betrachten.

Die homöopathische Methode stellt andererseits ein mächtiges Korrektiv für all jene bereit, die darunter leiden, dass ihr Geist vom Geld als Denkform dominiert wird. Soweit mit dem Ähnlichkeitsprinzip wahre Heilungen zu bewirken sind, sollten vor allem Homöopathen sich selbst von Miasmen jeder Art, einschliesslich mentaler, befreien können.

Letztendlich könnte sich der oben vorgestellte Ansatz der Wissenschaftskritik als homöopathisch in einem sehr weiten (metaphorischen) Sinn erweisen, der mehr der buddhistischen oder hinduistischen Methode des Reduzierens von Illusionen ähnelt als der kausal-analytisch affirmativen Methode der konventionellen modernen Medizin. Er beruht auf der Annahme, dass spätgeborene Kinder einer spätkapitalistischen Ära ohnehin gezwungen sind, von ihrem verwirrten Geist ausgehend, anzufangen zu denken - ganz in Analogie zu psorischen Patienten, von deren Lebenskraft ebenso angenommen wird, dass sie verstimmt sei. Wenn, nach Hahnemann, der verstimmten 
Lebenskraft ihr Feind (das Miasma) in Form eines leicht vergrösserten Bildes vorgehalten wird (in Form eines potenzierten Arzneimittels), wird sie dadurch veranlasst, «ihre Energie zu erhöhen» und das Miasma zu besiegen. Anstatt weiter beherrscht zu werden, erringt sie auf diese Weise ihre Souveränität zurück [43].

Analog müsste modernen fremdbestimmten Subjekten ihr vorherrschendes mentales Miasma (ihr Denken in der Geldform) vorgehalten werden, allerdings in kondensierter Form (d.h. auf den Begriff gebracht), um sie zu veranlassen, sich zu empören [44] und dagegen aufzulehnen - um sich auf diese Weise selbst von ihm zu befreien.

Wenn das passiert, würde der Homöopath wohl zusätzlich in den Stand gesetzt, auch die Homöopathie von parasitären ökonomischen Interessen und kapitalistischen Schemata - wie Innovationsdruck, Wettbewerb, Evaluationszwang, Benchmarking, Marketing usw. - zu befreien. Und so dürfte auf einer bewussten und wissenschaftlichen Ebene zu erkennen sein, dass die Homöopathie, im Unterschied zur konventionellen modernen Medizin, auch in Subsistenz-Wirtschaften praktiziert werden könnte, ganz ohne immer noch grössere monetäre Investments und Transaktionen auf masslos expandierenden Märkten.

\section{Schlussfolgerung und Ausblick}

Es scheint einiges darauf hinzudeuten, dass eine treue Nachfolge Hahnemanns mehr herausfordernde Konsequenzen haben kann als nur die Verschreibung homöopathischer Arzneimittel. Erstens mag Hahnemanns aufrichtiger und starker Charakter seine Nachfolger dazu inspirieren, selbst ein freies und unabhängiges Denken zu wagen. Zweitens mag eine umfassende Interpretation seines Ähnlichkeitsprinzips (mittels sämtlicher moderner Wissenschaften) sie zu einem ausgedehnten Studium der Bedingungen anleiten, die freies und vertrauensvolles Denken und Handeln begrenzen und beeinträchtigen. Und drittens, wenn sie diese Bedingungen schliesslich erkennen und Spielraum gewinnen, mögen Homöopathen sich selbst ebenso wie die Homöopathie befreien, deren Kern in der Vergangenheit durch monetäre Einflüsse in zunehmender Weise verwirrt worden ist.

Als eine damit einhergehende Nebenwirkung mag der wahre Hahnemannianer dabei auf einen Weg geraten, der zu der letzten Herausforderung des Lebens führt, die in der antiken griechischen Philosophie in Form des Imperativs «gnothi seauton» (erkenne dich selbst) bekannt war, was wiederum mit dem korrespondiert, was in den Upanishaden als die höchste Weisheit galt: zu wissen «tat twam asi» (das bist du).

\section{Disclosure Statement}

Keine Interessenkonflikte.

\section{Literatur}

$>1$ Albrecht H, Van Wijk R, Dittloff S: A new database on basic research in homeopathy. Homeopathy 2002;91:162-165.

$>2$ Ernst E: A systematic review of systematic reviews of homeopathy. Brit J Clin Pharmacol 2002;54:577-582.

3 Smith K: Against homeopathy - a utilitarian perspective. Bioethics 2012;26:398-409.

4 Weymayr C, Heissmann N: Die Homöopathie-Lüge. So gefährlich ist die Lehre von den weißen Kügelchen. München, Piper, 2012.

5 Schirrmacher F: Ego. Das Spiel des Lebens. München, Blessing, 2013.

6 Ehrenberg A: Das erschöpfte Selbst. Depression und Gesellschaft in der Gegenwart. Frankfurt/M., Suhrkamp, 2008.

7 Spaemann R: Schritte über uns hinaus. Gesammelte Reden und Aufsätze. 2 Bände. Stuttgart, Klett-Cotta, 2007-2010.

$>8$ Shang A, Huwiler-Müntener K, Nartey LL, et al: Are the clinical effects of homoeopathy placebo effects? Comparative study of placebo-controlled trials of homoeopathy and allopathy. Lancet 2005;366:726-732.
9 Vogt M: Teilchen, Wellen, Wahrscheinlichkeiten. Die Anfänge der Quantenmechanik. Göttingen, Cuvillier, 2011.

10 Maturana HR: Der Baum der Erkenntnis. Die biologischen Wurzeln des menschlichen Erkennens. München, Goldmann, 1990.

11 Chalmers AF: Wege der Wissenschaft. Einführung in die Wissenschaftstheorie, ed 6. Berlin, Springer, 2007.

12 Wieland W: Diagnose. Überlegungen zur Medizintheorie. Warendorf, Hoof, 2004.

13 von Weizsäcker V: Der Gestaltkreis. Theorie der Einheit von Wahrnehmen und Bewegen. Stuttgart, Thieme, 1947.

14 von Uexküll T: Theorie der Humanmedizin. Grundlagen ärztlichen Denkens und Handelns. München, Urban \& Schwarzenberg, 1988.

15 Egger JW: Das biopsychosoziale Krankheitsmodell. Grundzüge eines wissenschaftlich begründeten ganzheitlichen Verständnisses von Krankheit. Psychol Med 2005;16:3-12.

16 Schmidt JM: Thure von Uexkülls semiotisches Modell des Menschen als Grundlage für eine moderne Theorie der Homöopathie. Schweiz Z Ganzheitsmed 2012;24:233-241.
17 Berger PL, Luckmann T: Die gesellschaftliche Konstruktion der Wirklichkeit. Eine Theorie der Wissenssoziologie, ed 5. Frankfurt/M., Fischer, 1977.

18 Brodbeck KH: Die fragwürdigen Grundlagen der Ökonomie. Eine philosophische Kritik der modernen Wirtschaftswissenschaften. Darmstadt, Wissenschaftliche Buchgesellschaft, 2009.

19 Smith A: Der Wohlstand der Nationen. München, FinanzBuch, 2006.

20 Vogl J: Das Gespenst des Kapitals. Zürich, Diaphanes, 2010.

21 Brodbeck KH: Die Herrschaft des Geldes. Geschichte und Systematik, ed 2. Darmstadt, Wissenschaftliche Buchgesellschaft, 2012.

22 Unschuld PU: Ware Gesundheit. Das Ende der klassischen Medizin. München, Beck, 2009.

23 Maio G: Ärztliche Hilfe als Geschäftsmodell? Eine Kritik der ökonomischen Überformung der Medizin. Dtsch Ärztebl 2012; 109:A804-807.

24 Graeber D: Schulden. Die ersten 5000 Jahre. Stuttgart, Klett-Cotta, 2012. 
25 von Braun C: Der Preis des Geldes. Eine Kulturgeschichte. Berlin, Aufbau, 2012.

26 Bacon F: Instauratio magna. Londini, Billius, 1620.

27 Geissler, KA: Vom Tempo der Welt und wie man es überlebt. Freiburg i.Br., Herder 2004.

28 Maio G: Heilen als Management? Zum Verlust einer Kultur der verstehenden Sorge in Zeiten der Ökonomie. Z Allg Med 2012; 88:18-23.

29 Unschuld PU: Was ist Medizin? Westliche und östliche Wege der Heilkunst, ed 2 München, Beck, 2012.

30 Müller RW: Geld und Geist. Zur Entstehungsgeschichte von Identitätsbewusstsein und Rationalität seit der Antike. (Habilitationsschrift). Frankfurt/M., Campus, 1977.

31 Descartes R: Die Prinzipien der Philosophie. Lateinisch - Deutsch. Hamburg, Meiner 2005
32 Rauprich O, Steger F (Hrsg): Prinzipienethik in der Biomedizin. Moralphilosophie und medizinische Praxis. Frankfurt/M., Campus, 2005.

33 Spaemann R: Moralische Grundbegriffe. München, Beck, 2009.

34 Spaemann R: Über Gott und die Welt. Eine Autobiographie in Gesprächen. Stuttgart, Klett-Cotta, 2012.

35 Nietzsche F: Die fröhliche Wissenschaft. Aph. 125, vgl. 108, 343; in Nietzsche F: Sämtliche Werke. Kritische Studienausgabe in 15 Bänden. Band 3. München, DTV, 1980.

36 Schmidt JM: Die philosophischen Vorstellungen Samuel Hahnemanns bei der Begründung der Homöopathie (bis zum Organon der rationellen Heilkunde, 1810). München, Sonntag, 1990.

37 SchmidtJM:DieHomöopathie Hahnemanns zwischen Dogmatik und Dynamik; in Zurück zu den Wurzeln. 200 Jahre Homöopathie. Jubiläumskongress, Luzern,
1.-2.11.1996. Küssnacht am Rigi, Omida, 1997, pp 15-29.

38 Schmidt JM: Die Homöopathie im Span nungsfeld zwischen aristotelischer und moderner Wissenschaft. Schweiz Z Ganz heitsmed 2009;21:105-111.

39 Schmidt JM: Hahnemanns Homöopathie zwischen rationaler Heilkunde und Heilkunst. Schweiz Z Ganzheitsmed 2011; 23:224-232.

40 Unschuld PU: Der Arzt als Fremdling in der Medizin? Standortbestimmung, ed 2. Germering, Zuckschwerdt, 2010

41 Jütte R: «Und es sammelte sich ohne Verdruss von Seiten des Kranken in des Arztes Beutel» - Samuel Hahnemann und die Honorarfrage. MedGG 1999;18:149-167.

42 Aristoteles: Politik. Berlin, Akademie, 2011.

43 HahnemannS:Diechronischen Krankheiten ihre eigenthümliche Natur und homöopathische Heilung, ed 2. Band 4. Düsseldorf Schaub, 1838, p VIII.

44 Hessel S: Empört Euch! Berlin, Ullstein, 2011. 